\title{
Low Adhesion Braking Dynamic Optimisation for Rolling Stock (LABRADOR) Simulation Model
}

\author{
Hamid Alturbeh*, Julian Stow*, Alan Lawton ${ }^{\dagger}$ \\ *University of Huddersfield, UK h.alturbeh;j.m.stow@hud.ac.uk, ${ }^{\dagger}$ RSSB,UK alanlawton@msn.com
}

Keywords: Low adhesion, Train brake system.

\begin{abstract}
This paper describes the current version of the LABRADOR simulation tool that can predict the train brake system performance and support decision-making in the design and optimisation of the braking system including WSP, sanders, and the blending and control of friction and dynamic brakes. The model has been developed in MATLAB/SIMULINK and is intended to mimic the braking performance of both older and newer generations of multiple unit passenger trains. It provides a quantitative simulation tool to test different designs and support the optimisation of the brake systems for contemporary and future trains.
\end{abstract}

\section{Introduction}

Low adhesion in the wheel/rail interface can cause both safety and performance issues. In braking it can lead to station overruns and signals passed at danger (SPADs) and in traction it can lead to costly delays. Low adhesion can result from a number of causes including wet-rail, leaves, oil, etc. [1]. Modern rolling stock braking under low adhesion uses a combination of wheel slide protection (WSP) and sanding systems to increase adhesion in the wheel-rail contact in an attempt to avoid wheel damage and/or flats and to minimise braking distance.

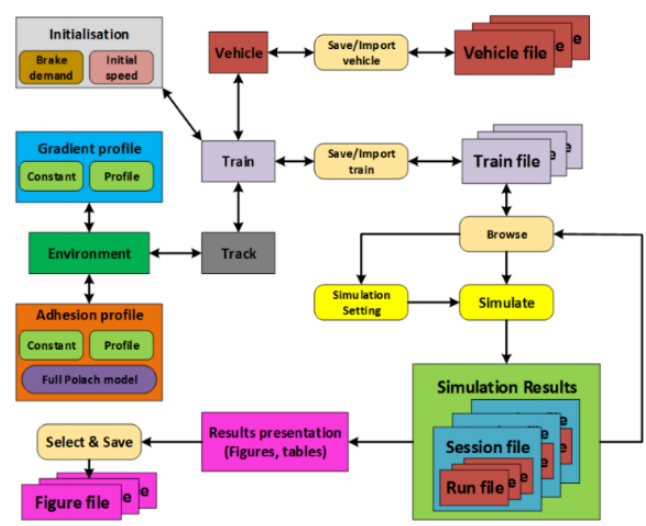

Fig. 1 Main LABRADOR model functions [3].

Over the years, research by British Rail Research, other organisations [2] [3] and more recently RSSB [4] [5] have contributed to a better understanding of low adhesion phenomena. Other studies have focused on the modelling of train braking systems [6]. Nevertheless, long train slide events still occur and the ability of modern simulation software to model the complex interdependence of brake system components in the overall behaviour of the brake system provides an opportunity to further understand, and even improve the braking performance of trains.

The aim of LABRADOR project is to develop simulation software capable of modelling the behaviour of modern multiple unit passenger trains braking under normal and low adhesion conditions by representing the complex interdependence of brake system components in the overall behaviour of the brake system. It is modular and allows easy specification of vehicle, bogie and wheelset subsystems. Within the wheelset subsystem WSP, sander, contact patch and temperature and adhesion subsystems exist. Figure 1 illustrates the functions within the LABRADOR simulation tool.

\section{LABRADOR Architecture}

The LABRADOR software presents four train models; single car, two, three, and four cars train models. Each of these models is assembled from a set of modules that simulate the behaviour of discrete functions within the braking system of a train. Some modules simulate physical features that can be found on each vehicles. For example, The WSP module, mechanical behaviour of wheelsets and the influence of sand on adhesion. Some modules simulate control functions such as the brake blending controller.

The outer layer of the model, the environment layer, will provide the inputs to a train module; initial speed and brake demand, adhesion and gradient etc. The train module contains one, two, three, or four functionally identical vehicle modules, each vehicle module contain a number of functionally identical wheelset modules. Each module type is functionally identical and provides the easiest way to exploit the duplicated systems within real, long trains. For example, changing the WSP system working for every vehicle in a 4 car train will involve change to just the WSP module that is replicated many times within the model structure for a long train. The modular structure of LABRADOR consists of a train module that contains single, two, three, or four vehicles modules. Each vehicle module contains four wheelset modules that each contains: one dynamic brake module; one WSP and friction brake module (WSP\&FB); one sander module; and one wheel module that contains a contact patch module.

The following sections describes the LABRADOR train models and the modules that make up the train models.

\subsection{Environment layer}

The environment layer is the top layer of the model which: 
1. Provides the external data required by the train module.

2. Allows the operator to set initial conditions for train position, train speed and drivers brake demand.

3. Passes initial condition information to the train module.

4. Takes data from the contact patch modules on the adhesion behind the wheelset to update the adhesion map.

5. Provides data to the contact patch modules on incoming adhesion for each wheelset.

6. Provides gradient information to allow calculation of gravitational forces by the vehicle module.

\subsection{Train module}

The train module consists of up to four vehicles and is the module that interacts with the environment layer as shown in Figure 2. The train module functions are:

1. Calculates the drag forces as a consequence of train speed and train gravitational forces using gradient data from environment.

2. Computes train acceleration, speed and position as a consequence of the drag, gravitational and wheel-rail forces applied to the train.

3. Allocates drivers brake demand, from the environment, between each vehicle, according to the state of brake equipment on the vehicle (dynamic brake isolation, WSP activity etc.) and on the make-up of the vehicles (is dynamic barking available on all vehicles, etc..).

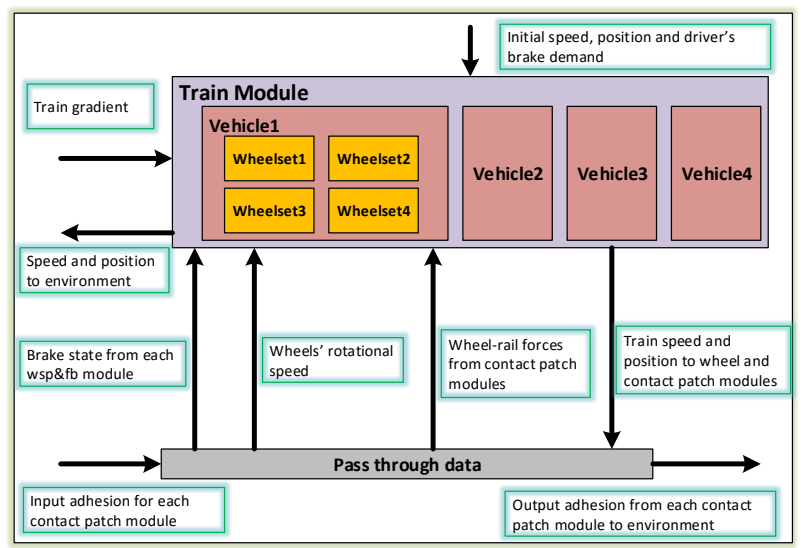

Fig. 2 Train module interaction with the environment layer

\subsection{Vehicle module}

A vehicle module contains four wheelsets modules as shown in Figure 2. The vehicle module: allocates vehicle brake demand as a combination of friction brake demand and dynamic brake demand to each wheelset, depending on the state of the vehicle's brake equipment; and calculates the load transfer due to train acceleration, wheel-rail forces and track gradient.

\subsection{Wheelset module}

The wheelset module groups a number of discrete functions as can be seen in Figure 3 that are: dynamic brake module; WSP and friction brake module (WSP\&FB); sanding control module; rotating wheelset module (wheel), within which is found the contact patch module. These functions are contained within the wheelset module because their actions are exclusively centred on one individual wheelset. The wheelset module has no specific function; it exists to contain the modules listed above and to receive certain data from the vehicle module, such as friction brake demand, dynamic brake demand, and train/vehicle and pass it to its inner modules.

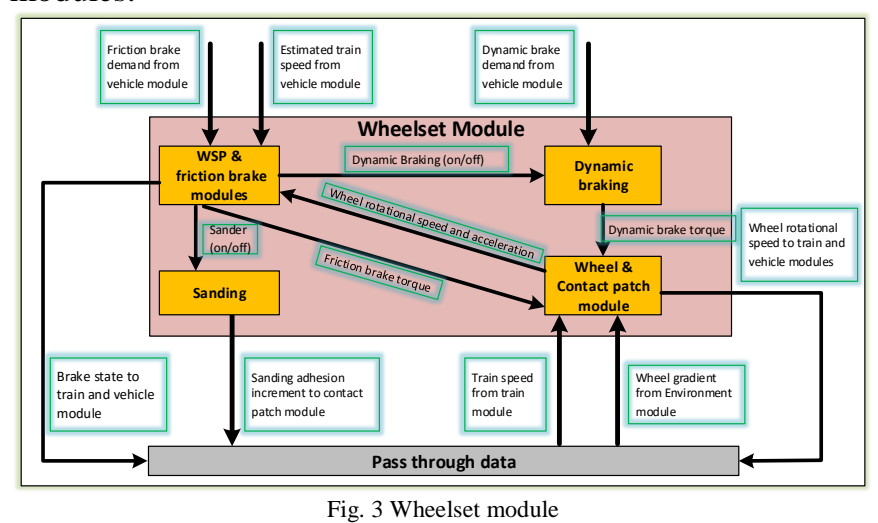

\subsection{Contact patch module}

The contact patch module: calculates the force in the contact patch and wheel slide as a function of wheelset rotational speed, train speed, adhesion at the contact patch and the force demand on the wheel-rail contact point; calculates the output adhesion; and computes the contact patch temperature as a consequence of adhesion, normal load and actual wheel slide.

\subsection{Dynamic brake module}

If enabled, the dynamic brake module produces a torque on the wheelset. This brake can be disabled if wheel slide is excessive; dynamic brake torque is then zero until the train stops. The dynamic brake torque is proportional to the dynamic brake demand, and there is negligible delay between changes in dynamic brake demand and dynamic brake torque.

\subsection{Wheel slide protection \& friction brake module.}

The activities of the WSP and friction brake are closely linked, hence, they are modelled as one module. The (WSP\&FB) module controls braking and sanding as a consequence of wheel slide $(w s)$ :

1. Receives wheel rotational acceleration and speed from the wheel module.

2. Calculates wheel slide using the actual wheelset rotational speed, wheel radius and the chosen train speed.

3. Controls friction brake torque taking into consideration delays in the pneumatic system.

4. Activates wheelset sanding and isolates the dynamic brake, depending on wheel slide.

5. Describes the state of the brake equipment (dynamic brake enabled/disabled, friction brake on/off, sanding on/off) to the vehicle module for use in setting brake demands 


\subsection{Sander module}

Sand is applied to the wheel-rail contact patch when wheel slide exceeds a certain level. The WSP\&FB module monitors wheel slide and signals to the sander module to increase adhesion, subject to a time lag. The sander module tells the contact patch module that input adhesion is increased.

\subsection{Wheel module}

This module models the rotational behaviour of the wheelset, depending on the dynamic and friction brake torques, the wheel load and the contact patch behaviour. The wheel module will: calculate drag force for the wheelset; calculate gravitational force for the wheelset; calculate the force demand on the wheel-rail contact point and relate gravitational force, drag force, friction brake torque and dynamic brake torque (the force demand on the contact point) and available wheel-rail force to calculate wheel rotational acceleration and speed.

\section{Train brake model}

Any train braking model should quantify two main quantities and their associated derivatives:

a. The train, vehicle and wheelset positions: By defining $x(t)$ as the train position at a particular time $t$ (in $\mathrm{m}$ ) and by defining $x_{v}(t)$ as the longitudinal position for each vehicle and defining $x_{v j}(t)$ for each wheelset in each vehicle at a particular time $t$, where $v=1,2,3,4$ is the vehicle index, and $j=1,2,3,4$ represents the wheel index. The train position is assumed to be equal to position of the first wheelset at the first vehicle, i.e. $x(t)=x_{11}(t)$. The vehicles' positions $x_{v}(t)$ are the position of the first wheelset in that vehicle which can be directly defined from the train position by the following:

$$
x_{v}(t)=x(t)+\left(V_{n o}-1\right)\left(V_{l}+V_{s}\right)
$$

Where $V_{n o}$ is the vehicle number $V_{l}$ is vehicle length and $V_{s}$ is the vehicle spacing.

The wheelsets' position $x_{v j}(t)$ are directly defined from the vehicle position $x_{v}(t)$ as follows:

$$
\begin{gathered}
x_{v 1(t)}=x_{v}(t) ; x_{v 2}(t)=x_{v}(t)+W_{s} \\
x_{v 3}(t)=x_{v}(t)+B_{s} ; x_{v 4}(t)=x_{v}(t) \\
+W_{s}+B_{s}
\end{gathered}
$$

where $W_{s}$ and $B_{s}$ are the wheelset and the bogie spacing. The geometric centre of the vehicle is defined as:

$$
x_{v G}(t)=x_{v}(t)+\frac{1}{2}\left(W_{S}+B_{S}\right)
$$

for calculating the effect of track gradient $\left(i=i\left(x_{v G}(t)\right)\right)$.

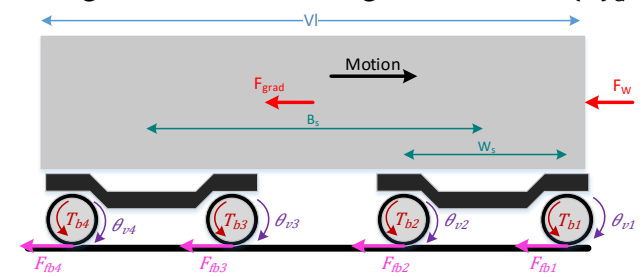

Fig. 4 Schematic of the first vehicle.

b. Wheelset rotations: By defining $\theta_{v j}(t)$ as the wheelset rotation for vehicle $v$ wheelset at a particular time $t$ (in rad).
Figure 4 shows a schematic of the first vehicle with the longitudinal and rotational degrees of freedom $\left(x_{v}(t), \theta_{v 1}(t)\right.$, $\theta_{v 2}(t), \theta_{v 3}(t)$ and $\left.\theta_{v 4}(t)\right)$ and some longitudinal dimensions (bogie and wheelset spacing $B_{s}, W_{s}$, and vehicle length $V_{l}$ ).

\subsection{Equations of motion}

The degrees of freedom $x(t)$ and $\theta_{v j}(t)$ are computed through numerical integration using as boundary conditions: known initial speeds $\dot{x}_{v}(t=0)=\dot{x}_{0}$ for the vehicle and $\dot{\theta}_{v j}(t=0)=\dot{x}_{0} / R$ for all wheelsets. The longitudinal and rotational equations are defined to conduct numerical integration.

\subsubsection{Longitudinal dynamics}

For simplicity a single vehicle model is considered in this section. Three different longitudinal forces are applied to the vehicle:

1. The wheel-rail forces due to friction in the contact between the wheel and the rail $\left(F_{f b_{j}}(t)\right.$ for all wheelsets);

2. The train drag forces $\left(F_{w}(t)\right)$;

3. The horizontal component of the weight due to the track gradient $\left(F_{\text {grad }}(t)\right)$;

Equation (5) sums all the applied forces and divides them by the vehicle mass $(M)$ to compute the longitudinal vehicle acceleration $(\ddot{x}(t))$.

$$
\ddot{x}(t)=\frac{1}{M}\left(-F_{w}(t)-F_{\text {grad }}(t)-\sum_{j=1}^{j=4} F_{f b_{j}}(t)\right)
$$

\subsubsection{Rotational dynamics}

Two torques are applied in each wheelset $j$ :

1 . The torque due to the wheel-rail force $F_{f b}(t)$ applied in

the contact between the wheel and the rail with a moment arm equal to the Radius $(R)$;

2. The braking torque $T_{b_{j}}(t)$;

Equation (6) sums all the applied torques and divides them by the wheelset rotational inertia $(J)$ to compute the wheelset rotational acceleration $(\ddot{\theta}(t))$.

$$
\ddot{\theta}_{j}(t)=\frac{1}{J}\left(+F_{f b_{j}}(t)-T_{b_{j}}(t)\right)
$$

Figure 5 provides a schematic representation of the torques applied to each wheelset $\left(F_{f b}(t) . R\right.$ and $\left.T_{b_{j}}(t)\right)$. The wheelrail force is calculated in the adhesion model.

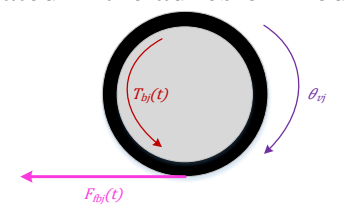

Fig. 5 Wheelset diagram representing the torques applied to each wheelset.

\subsection{Adhesion model}

The adhesion model is based on a method developed by Polach [7] for calculation of creep forces in multi-body 
simulation. The model is based on a theoretical model for longitudinal and lateral creep assuming a coefficient characterising the contact shear stiffness. The magnitude of the resultant creep force $F$ is obtained by integrating the shear stress distribution over the contact surface. The creep force components $F_{x}, F_{y}$ are assumed to be proportional to the longitudinal and lateral creepages. The contact area is assumed to be constant elliptical shape with half-axes $a, b$ and normal stress distribution according to Hertz. Figure 6 shows the distribution of the tangential stress $\tau$

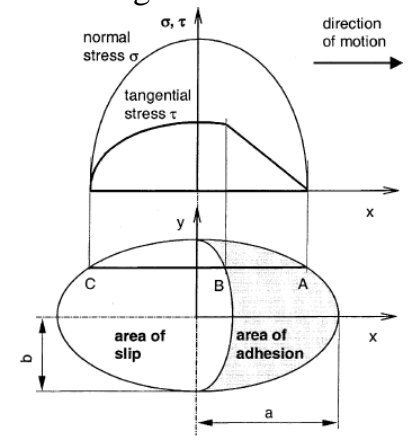

Fig. 6 Distribution of normal and tangential stresses in the wheel-rail contact [7] . The maximum value of tangential stress at any arbitrary point is given by:

$$
\tau_{\max }=\mu \sigma
$$

Where $\sigma$ is the normal stress and $\mu$ is the coefficient of friction. The friction coefficient depends on the slip velocity where the friction coefficient decreases with increasing slip (creep) velocity between wheel and rail. The following equation expresses the variable friction coefficient:

$$
\mu=\mu_{0}\left[(1-A) e^{-B w}+A\right]
$$

Where $\omega$ is the total creep (slip) velocity, $B\left[s . m^{-1}\right]$ is the coefficient of exponential friction decrease, and $A$ is the ratio friction coefficients $A=\frac{\mu_{\infty}}{\mu_{0}}$, where $\mu_{\infty}$ is the friction coefficient at infinite slip velocity and $\mu_{0}$ is the maximum friction coefficient. The longitudinal tangential creep force (without spin) is given as follows:

$$
\begin{gathered}
F_{x}=\frac{2 Q \mu}{\pi}\left(\frac{k_{A} \varepsilon_{x}}{1+\left(k_{A} \varepsilon_{x}\right)^{2}}+\operatorname{atan}\left(k_{S} \varepsilon_{x}\right)\right) ; \\
\varepsilon_{x}=\frac{G \pi a b c_{11}}{4 Q \mu} s_{x}
\end{gathered}
$$

Where $G$ is shear module ( $G=8.4 .10^{10}$ for steel), $c_{11}$ is coefficient from Kalker's linear theory [8], and $s_{x}$ is the longitudinal creep. The adhesion coefficient is:

$$
f_{x}=\frac{F_{x}}{Q}
$$

Polach has used different reduction factors $k_{A}$ in the area of adhesion and $k_{S}$ in the area of slip to model the different railwheel condition $\left(k_{S} \leq k_{A} \leq 1\right)$. The lateral components are neglected in the LABRADOR model.

\subsection{External forces model}

Two external forces that are considered during braking: 1. Frictional and aerodynamic drag forces: The Davis equation is used to calculate train drag forces as a second order polynomial of train speed [9].

$$
F_{w}(t)=A+B \cdot \dot{x}(t)+C \cdot(\dot{x}(t))^{2}
$$

Equation (12) gives an empirical expression depending on the vehicle speed $(\dot{x}(t))$ and coefficients $A, B$ and $C$. Coefficients $A, B$ and $C$ for different trains have been estimated based on expressions developed by [9], and [10].

2. The horizontal component of train weight due to the track gradient: due to the track gradient, the weight of the vehicle may have a horizontal component in the longitudinal direction contributing to acceleration on a falling gradient or braking of the train on a rising one. Equation (13) gives the expression for the horizontal component of the weight $(M . g)$ due to the track gradient $i(t)$.

$$
F_{\text {grad }}(t)=M \cdot g \cdot i(t)
$$

If it is a horizontally straight segment, then $i(t)=0$ and $F_{\text {grad }}(t)=0$. The track gradient $i=i(t)=i\left(x_{G}(t)\right)$ will be computed using the longitudinal position of the geometric centre of the vehicle $x_{G}(t)$ which is given by Equation (4).

\subsection{Driver's brake controller}

The driver's brake controller is able to mimic a standard 4step brake controller; notch 1, 2, 3 and Emergency, corresponding to values of train brake demand of $3 \%, 6 \%, 9 \%$ and $12 \%$ of gravitational acceleration $g$. A brake demand subsystem in LABRADOR can generate constant and variable values of driver brake demand.

\subsection{Wheel sild protection system (WSP)}

LABRADOR uses a simple model of WSP in which it is assumed that the WSP controller has perfect knowledge of vehicle speed. The WSP model assumes two possible positions: apply and release. These two actions are taken based on the wheel slide $s_{j}(t)$ for wheelset $j$ at a particular time. A WSP system would then control the speed difference or wheel slide for each wheelset and would apply or release the brakes depending on these terms. Therefore if the wheel slide is greater than a certain threshold for more than a certain time threshold, then the WSP is activated and the brakes are released, until wheel creepage is less than a certain threshold for more than a certain time threshold, whereupon the WSP is deactivated and the brake is re-applied to the demanded level after a delay time.

\subsection{Sanding system model}

The sanding model in LABRADOR uses the model that developed by Alan Lawton [11] which is based on a fixed rate of $2 \mathrm{~kg} /$ minute. The sander is activated and deactivated based on the creepage value. For example, the sander is triggered if wheel slide is higher than a certain threshold, which can be defined by the user, for a certain amount of time. The sander will be deactivated as soon as the creepage value become less than a certain threshold. The change in adhesion due to sand at each wheelset is calculated based on [11] in which the adhesion boost from sand is about 0.06 and the residual sand ratio is $50 \%$. However, the user can configure these values with other sanding system parameters Figure 7 shows simulation results for a two car train operating in low adhesion conditions with a sander fitted on the third 
wheelset. It can be seen that when the sander is triggered the adhesion increases by the amount that equals to the maximum adhesion increment due to sanding, which is 0.06 in this case. Then the adhesion boost for the following wheelset is reduced by $50 \%$ at each wheelset.

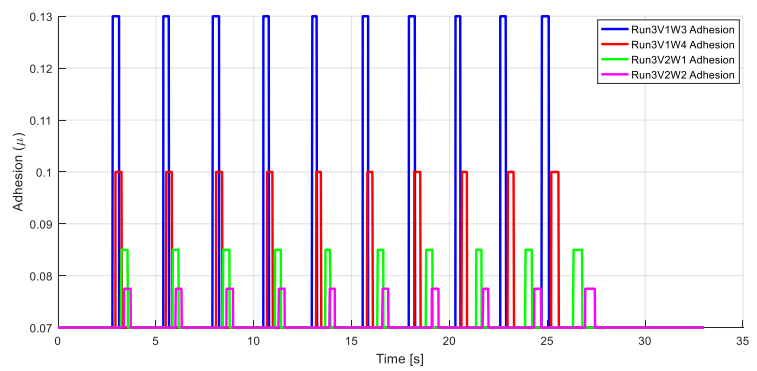

Fig. 7 Adhesion boost due to sanding effect for wheelset3 (blue), wheelset 4 (red), wheelset5 (green), and wheelset6 (magenta)

\subsection{Dynamic braking and brake blending model}

According to [12], brake blending is the automatic mixture of the dynamic braking provided by the traction system and the traditional friction braking system.

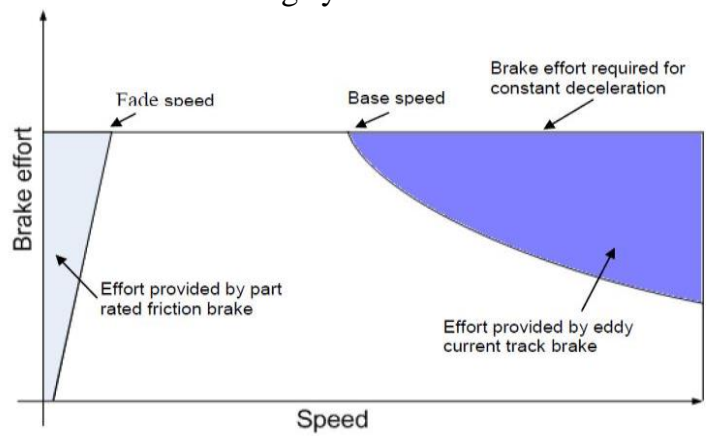

Fig. 8 Typical dynamic brake effort curve [13]

The dynamic brake uses the traction motors as generators, producing energy that can be dissipated in the form of heat (rheostatic braking) or fed back into the overhead line or third rail (regenerative braking). Figure 8 describes the dynamic brake effort for different speeds. Given the fade and base speeds ( $S_{\text {fade }}$ and $S_{\text {base }}$ ), three speed regions can be distinguished:

- $\quad\left(v<S_{\text {fade }}\right)$ the dynamic brake effort 'fades' linearly (decreases linearly) with speed;

- $\quad\left(S_{\text {fade }} \leq v<S_{\text {base }}\right)$, the dynamic brake effort is at most the maximum dynamic force $\left(F_{-}(\max\right.$, dynamic $\left.)\right)$, with zero brake force from other brake systems;

- $\quad\left(v \geq S_{\text {fade }}\right)$, the brake effort reduces with speed squared to a minimum of $\alpha . F_{-}$(max, dynamic) when the train speed is equal to the top/design speed $\left(\dot{x}=S_{\text {top }}\right)$, in which $\alpha$ is a positive quantity that reflects the amount of the maximum braking force due to dynamic braking at the top speed.

The distribution of brake demand into the dynamic brake demand component and the friction brake demand component depending on the train speed (or estimated train speed) can be defined by the user. It is assumed that the remaining brake effort is supplied by the friction brakes. Nevertheless, it is important to mention that this distribution of brake effort can be done locally for each motored wheelset or in more sophisticated ways (e.g. at each vehicle or at each unit).

\subsection{Contact patch temperature model}

The contact patch temperature model was developed based on [14] in which the contact patch temperature due to slip in braking is given by:

$$
\theta_{w}=\frac{2.26 \times P_{m} \cdot \mu}{K}\left(\frac{a . \alpha \cdot V}{\pi}\right)^{1 / 2}\left\{1-(1-s)^{1 / 2}\right\}
$$

Where $\theta_{w}$ is the interface temperatures between the wheel and the rail, $P_{m}$ is the maximum contact pressure, $\mu$ is the friction coefficient between the wheel and the rail, $K$ is the thermal conductivity (for steel $K=46 \mathrm{~W} \cdot \mathrm{m}^{-1} \cdot{ }^{\circ} \mathrm{C}^{-1}$ ), $a$ is the semi-axis of the contact ellipse in the longitudinal direction, $\alpha$ is the thermal diffusivity (for steel $\alpha=0.12 \times 10^{-4} \mathrm{~m}^{2} . \mathrm{s}^{-1}$ ), $V$ is the vehicle speed and $s$ is the slip ratio.

Note that the maximum pressure $P_{m}$ depends on the normal load $(N)$ and semi-axis dimensions of the contact patch ( $a$ and $b)$ and can be computed as:

$$
P_{m}=\frac{3 . N}{2 \pi a b}
$$

\section{Model validation}

This is a limited validation based on historic brake tests under 'high' adhesion conditions (i.e. no wheel slide) reported by BR in 1991 [15] [16] [17] [18].

Class 153: A series of on-track braking tests are reported in BR report [15] for a single car class 153 diesel unit. Several simulations were run with different initial braking speeds and using the full Polach model for dry conditions. All the simulation results are plotted in Figure 9 and they are compared with experimental tests. It can be seen that at low speeds there is a good match between predicted performance and practice. However, for higher speeds, the LABRADOR model underestimates the braking distance for the tread braked class 153. Whilst this might be due to the fact that the pneumatic system is not being directly modelled in LABRADOR, a plausible explanation is that that tread brakes are more susceptible to brake fade (due to heating of the blocks) on stops from higher speeds.
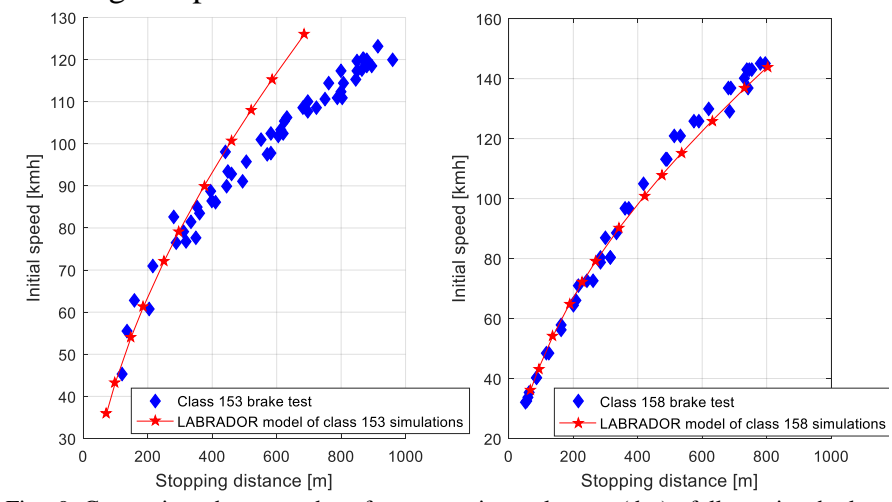

9 Comparison between data from experimental tests (dry), full service brake application (NOTCH 3 ) and simulations from LABRADOR single vehicle braking model for different initial speed for train class 153 (left) and class 158 (right)

Class 158: The class 158s are disc braked DMUs with a maximum speed of $90 \mathrm{mph}$. A series of on track braking tests 
are reported in BR for a 2-car class 158 diesel multiple unit [16] [17] [18].

Simulation results for the LABRADOR Class 158 model (in dry conditions) are compared with the experimental tests (also in dry conditions) in Figure 9 for crush laden conditions. It can be seen that LABRADOR simulation results of Class 158 show a much better fit with the experimental data than the simulation results for Class $153 \mathrm{~s}$ as the brake pads for disc break show much less variability than for the brake block material used on class $153 \mathrm{~s}$.

\section{Graphical User Interface (GUI)}

The LABRADOR model allows the user to define the parameters of the different modules by using a Graphical User Interface (GUI), allowing manual configuration of vehicle, train, environment, and simulation specific inputs, through a set of menus, toolbars, push-buttons and list boxes. Input screens contain default data values in order to minimise the time required for model set-up. Inputs are grouped in panels as seen in Figure 11.

After setting the parameters and configuring the subsystems, users can initiate the simulation and then the outputs can be presented by using set of flexible figures layout.

The generated and stored outputs include train speed, train acceleration, wheel-rail force, wheel rotational speed, wheel load, adhesion, gradient, wheel-rail force/load, WSP status, sander status, creepage, contact patch temperature, friction brake demand, and dynamic brake demand.

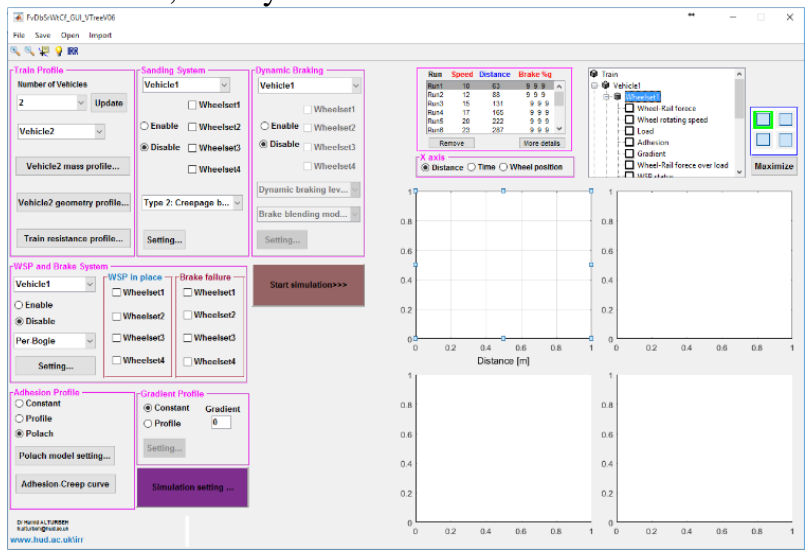

Fig. 11 LABRADOR Graphical User Interface (GUI)

\section{Conclusion}

The LABRADOR train braking model provides a basis for simulation and assessment of alternative braking system configurations for different trains under varying track gradient and adhesion profiles. The model is configured to preserve the modularity of the various sub-systems within the braking system. Each sub-system is modelled separately in MATLAB/SIMULINK. This approach enables the model to be extended to represent longer trains and also to model the various brake system architectures present in older, contemporary and future rolling stock. LABRADOR will allow the study of specific brake control features such as WSP strategies, sanding effectiveness, dynamic brake utilisation, traction performance, etc. This understanding will help train operators, maintainers and integrators to optimise the braking performance of their trains.

\section{Acknowledgements}

The LABRADOR project has been undertaken as part of the Strategic Partnership between RSSB and the University of Huddersfield.

\section{References}

[1] L. Buckley-Johnstone, R. Lewis, K. Six and G. Trummer, "Modelling and quantifying the influence of water on wheel/rail adhesion levels," RSSB, 2016.

[2] ORE, "Adhesion during Braking and Anti-skid Devices, ORE B 164 , RP 1, Synthesis of Current knowledge concerning adhesion,", Office for Research and Experiments of the International Union of Railways, Utrecht, 1985.

[3] ORE, "Summary of theoretical and practical knowledge of poor adhesion conditions acquired since publication of B 164/RP 2," Office for Research and Experiments of the International Union of Railways, Utrecht, 1992

[4] RSSB, " Review of Low Adhesion Research - Final report (T354).," RSSB, London, 2004.

[5] RSSB, “Benefits of all-electric braking. Final Report (T860).," RSSB London, 2012.

[6] L. Pugi, M. Malvezzi, S. Papini and G. Vettori, "Design and preliminary validation of a tool for the simulation of train braking performance," Journal of Modern Transportation, vol. 21, no. 4, pp 247-257, 2013

[7] O. Polach, "Creep forces in simulations of traction vehicles running on adhesion limit," Wear, pp. 992-1000, 2005.

[8] J. J. Kalker, "A fast agorrithm for the simplified theory of rolling contact," Vehicle System Dynamic, pp. 1-13, 1982.

[9] B. P. Rochard and F. Schmid, "A review of methods to measure and calculate train resistances.," Proceedings of the Institution of Mechanical Engineers, Part F: Journal of Rail and Rapid Transit, pp. 185-199, 2000

[10] D. S. Armstrong and P. Swift, "Lower energy technology. Part A, identification of energy use in multiple units. Report MR VS 077,," British Rail Research, Derby, 1990.

[11] A. Lawton, "The Effect of Sanders in Improving Train Braking Performance When Adhesion is Low," in The Sephenson Conference: Research for Railways, London, 2017.

[12] A. L. Nicholas, "Braking systems and their control architectures," in IET Professional Development Course on Electric Traction Systems, London, 2012.

[13] RSSB, "Benefits of all-electric braking, Final Report (T860)," 2012.

[14] M. A. Tanvir, "Temperature rise due to slip between wheel and rail- an analytical solution for Hertzian contact.,"Wear, pp. 295-308, 1980.

[15] BR, “ British Rail Report No. 1278G - Class 153 Type tests - Braking performance.," BR, 1991.

[16] BR, “British Rail Report No. 1159C - Class 158 Type tests - Full Service Braking performance of unit 158701 tare, seated and crush laden.," BR, 1991.

[17] BR, "British Rail Report No. $1159 \mathrm{~K}$ - Class 158 Type tests - Full Service Braking performance and holding capacity of step 1 brake on unit 748.," BR, 1991.

[18] BR, “ British Rail Report No. 1159M - Class 158 Brake Tests - Full Service Braking of unit 797 with alternative friction materials.," BR, 1991.

[19] RSSB, "Understanding the current use of sanders on multiple units (T796). Final report and Appendices," RSSB, 2010 